\title{
Progress on development of the new FDIRC PID detector
}

J. Va’vra ${ }^{\mathrm{k}, *}$, N. Arnaud ${ }^{\mathrm{f}}$, A. Yu. Barnyakove, M. Yu. Barnyakove, C. Beigbeder ${ }^{\mathrm{f}}$, M. Benettoni ${ }^{\mathrm{g}}$, M. El Berni $^{\mathrm{f}}, \mathrm{M}^{\mathrm{e}}$ Borsato ${ }^{\mathrm{f}}$,

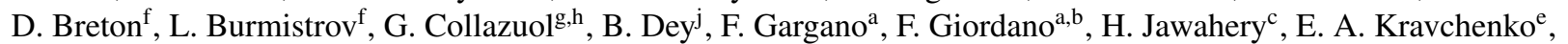
S. A. Kononov ${ }^{\mathrm{e}}$, H. Lebbolo ${ }^{\mathrm{i}}$, D. W. G. S. Leith ${ }^{\mathrm{k}}$, F. Loparco ${ }^{\mathrm{a}, \mathrm{b}}$, M. N. Mazziotta ${ }^{\mathrm{a}}$, J. Maalmi-Di Bello ${ }^{\mathrm{f}}$, K. Nishimura ${ }^{\mathrm{d}}$,

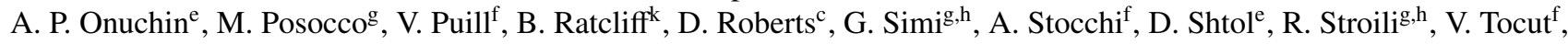
E. Twedt ${ }^{\mathrm{c}}$, G. S. Varner ${ }^{\mathrm{d}}$

${ }^{a}$ INFN Sezione di Bari, I-70126 Bari, Italy

${ }^{b}$ Dipartimento di Fisica "M. Merlin", Università di Bari,I-70126 Bari, Italy

${ }^{c}$ University of Maryland, College Park, Maryland 20742, USA

${ }^{d}$ University of Hawaii, Honolulu, 96822, USA

${ }^{e}$ Budker Institute of Nuclear Physics, Novosibirsk 630090, Russia

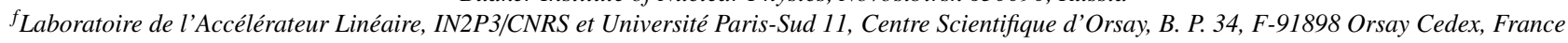
${ }^{g}$ INFN, Sezione di Padova, I-35131 Padova, Italy

${ }^{h}$ Dipartimento di Fisica e Astronomia "G. Galilei, Universit di Padova, I-35131 Padova, Italy

${ }^{i}$ Laboratoire de Physique Nucléaire et de Hautes Energies, IN2P3/CNRS, Université P. et M. Curie-Paris6, Université D. Diderot-Paris7, F-75252 Paris, France ${ }^{j}$ University of California at Riverside, Riverside, California 92521, USA

${ }^{k}$ SLAC National Accelerator Laboratory, Stanford, California 94309 USA

\begin{abstract}
We present a progress status of a new concept of PID detector called FDIRC, intended to be used at the SuperB experiment, which requires $\pi / \mathrm{K}$ separation up to a few $\mathrm{GeV} / \mathrm{c}$. The new photon camera is made of the solid fused-silica optics with a volume $25 \times$ smaller and speed increased by a factor of ten compared to the BaBar DIRC, and therefore will be much less sensitive to electromagnetic and neutron background.
\end{abstract}

Key words: Particle identification detectors, DIRC, RICH detectors PACS: 29.40.Cs, 29.40.Gx

\section{Introduction}

The BaBar experiment has used very successfully the DIRC PID detector (DIRC = Detector of Internally Reflected Cherenkov light) [1]. The original DIRC had one weak point: its huge photon camera, filled with 6,000 liters of ultra-pure water, was sensitive to electromagnetic and neutron background. This needs to be improved as the SuperB luminosity will increase $100 \times$. Therefore we have exchanged this simple pinhole camera at the heart of the DIRC detector into a focusing camera with sophisticated, solid fused-silica optics, while shrinking it to $1 / 25$ th of its former size and increasing its speed by a factor of ten [2]. The new detector is called FDIRC (Focused Detection of Internally Reflected Cherenkov light). Each of the 12 FDIRC Photon cameras will have $48 \mathrm{H}-8500$ MaPMTs, providing excellent timing capability for single photons $\left(\sigma_{T T S} \sim 140 \mathrm{ps}\right.$, TTS is a transit time spread). It is also necessary to implement new front-end electronics (FEE) with much higher timing precision $\left(\sigma_{\text {Electronics }} \sim 100 \mathrm{ps}\right)$, higher hit rate capability (few $\mathrm{MHz}$ hit rate per pixel), and small dead time (less than $5 \%$ at $1 \mathrm{MHz}$ rate) [3]. These improvements will compensate for the increase in luminosity $(\times 100)$ and background between the two generations of experiments. A full

\footnotetext{
*Corresponding author

Email addresses: jjv@slac.stanford.edu (J. Va'vra )
}

scale FDIRC prototype module covering 1/12 of the barrel azimuth will be tested in the cosmic ray telescope (CRT) [4] at SLAC with 3D tracking using muons.

\section{FDIRC design and construction}

A very important design feature of FDIRC photon camera is to measure the Cherenkov angle with $\mathrm{x}-\mathrm{y}$ pixels alone (the detector coordinate system where the y-direction is vertical and the $\mathrm{x}$-direction is horizontal), i.e., a high resolution timing is not needed. Time is used, however, to cut the background, perform the chromatic correction by timing, and be part of PID maximum likelihood.

Figures 1 and 2 show the new FDIRC photon camera design. The camera was designed with one important constraint: it has to reuse the BaBar DIRC radiators (bar boxes). This reduced possible design choices. The optics was designed using a graphical manual ray tracing [5], then checked by computer ray tracing using Mathematica program, and then checked with MC simulation [6]. The photon camera consists of two parts: (a) a focusing block (FBLOCK) with cylindrical and flat mirror surfaces, and (b) a new wedge. The wedge at the end of the bar rotates rays with large transverse angles (in the focusing plane) before they emerge into the focusing structure. The old wedge is too short so that an additional wedge element must be added 


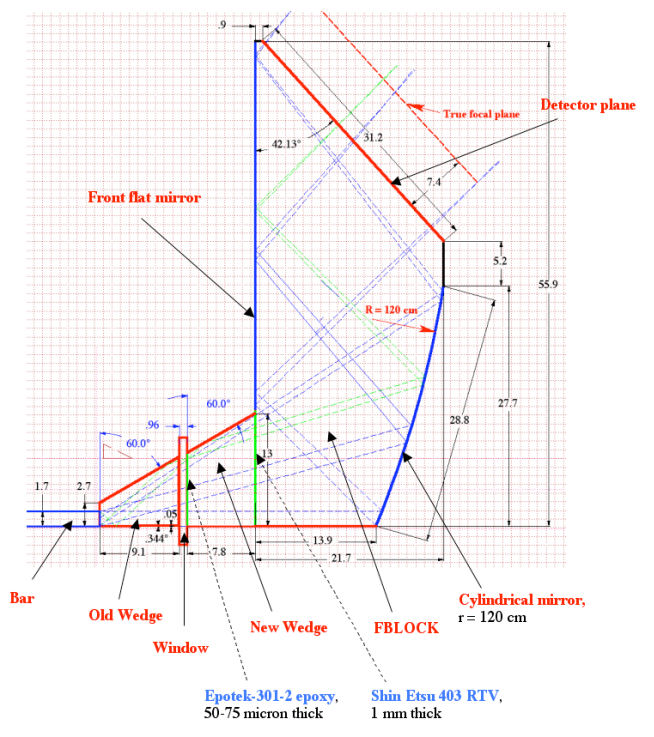

Figure 1: Optical design of FDIRC photon camera.

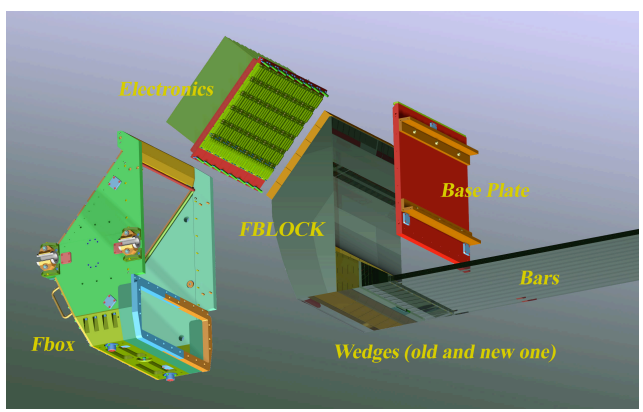

Figure 2: Parts of the FDIRC photon camera.

to insure that all rays strike the cylindrical mirror. The cylindrical mirror is rotated appropriately to make sure that all rays reflect onto the FBLOCK flat mirror, preventing reflections back into the bar box itself; the flat mirror then reflects rays onto the detector focal plane with an incidence angle of almost 90 degrees. The focal plane is located in a slightly under-focused position to reduce the FBLOCK size and therefore its weight. Precise focusing is unnecessary, as the finite pixel size would not take advantage of it.

There are several important advantages gained in moving from the BaBar pinhole focused design with water coupling to a focused optical design made of solid fused silica: (a) the design is modular; (b) sensitivity to background, especially to neutrons, is significantly reduced; (c) the pinhole-size component of the angular resolution in the focusing plane can be removed by focusing with cylindrical mirror, and timing can be used to measure the chromatic dispersion, thus improving performance; (d) the total number of photomultipliers is reduced by about one half compared to a non-focusing design with equivalent performance; (e) there is no risk of water leaks into the SuperB detector, and no time-consuming maintenance of a water system, as was required to operate BaBar safely.

Each new camera will be attached to its BaBar bar box with an optical RTV glue, which will be injected in a liquid form

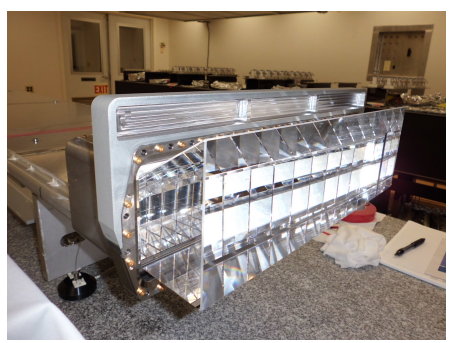

Figure 3: New wedge glued to the bar box window.

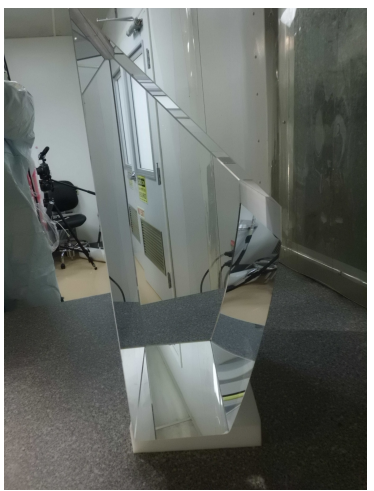

Figure 4: Finished FBLOCK, made of solid Corning 7980 fused silica.

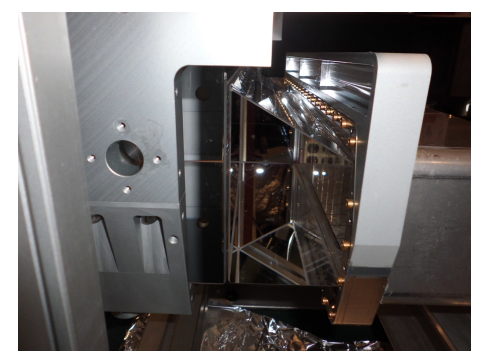

Figure 5: Details of coupling between FBLOCK, new wedge and bar box. A gap between FBLOCK and new wedge is filled with the RTV.

between the bar box window and the new camera and cure in place. As Fig. 1 shows, the cylindrical mirror focuses in the radial (y) direction, while pinhole focusing is used in the direction out of the plane of the schematic (the x-direction). Photons that enter the FBLOCK at large $\mathrm{x}$-angles reflect from the parallel sides, leading to an additional ambiguity. However, the folded design makes the optical piece small, and places the photon detectors in an accessible location, improving both the mechanics and the background sensitivity. Since a given photon bounces inside the FBLOCK only 2-4 times, the requirements on surface quality and polishing for the optical pieces are much less stringent than that required for the DIRC bar box radiator bars.

Figure 3 shows the new Wedge (glued to bar box), and Fig. 4 shows the FBLOCK. They are made of radiation hard fused silica (Corning 7980). The manufacturing was done in three steps: (a) grinding final shapes about 1-2 mm oversized, (b) polishing to final size and surface polish of better than $30 \mathrm{~A} \mathrm{rms}$, (c) coating two FBLOCK's reflecting surfaces with aluminum with $\mathrm{SiO}_{2}$ overcoat to protect it. Figure 5 shows the coupling 
between FBLOCK and new Wedge with a $1 \mathrm{~mm}$ thick RTV. This coupling can be cut with a razor wire enabling a possible separation of the photon camera from the bar box. Figure 6 shows the final FDIRC full size prototype located in CRT.

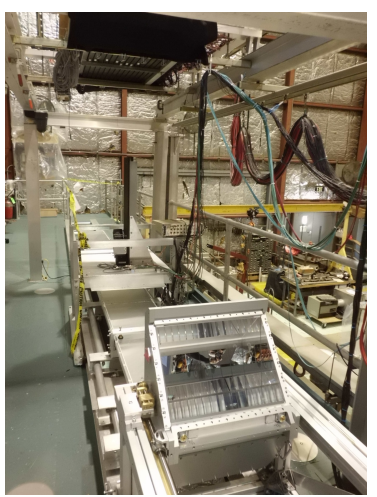

Figure 6: The photon camera and 5m-long bar box installed in the SLAC CRT. The picture shows the FBLOCK focal plane yet to be populated with detectors.

\section{Choice of photodetectors and electronics}

There were three Hamamatsu photon detectors under consideration, the H-8500 (64 pixels) and the H-9500 (256 pixels), and very recently R-11265-00-M64 (64 pixels) multianode PMTs (MaPMT) by Hamamatsu. At present, our nominal choice is a 12-stage H-8500 tube. It has a sufficient single electron timing spread $\left(\sigma_{T T S} \sim 140 \mathrm{ps}\right)$, it can be obtained with somewhat enhanced quantum efficiency QE of 24\%, it has more acceptable uniform gain response across its face (1:2.5), and it has been studied quite throughly (see, for example, [7] and [8]). Very recently another Hamamatsu tube, R-1126500-M64, came up for a consideration. Its main attractions are (a) super-bialkali QE of 36\%, (b) small $2.8 \mathrm{~mm}$ pixels, which would allow small binning in y-direction, and therefore better Cherenkov angle resolution, and (c) small dead space around tube boundaries. We are also considering a SiPMT-arrays, such as Hamamatsu C11206-0808FA, because of a small pixel size and high QE. However, its present noise performance is a concern, which could further increase if neutron doses would reach $10^{10} \mathrm{n} / \mathrm{cm}^{2}$.

With a H-8500 MaPMT choice, one photon camera would have 48 tubes, the entire FDIRC system 576 tubes and 18,432 pixels (here we assume that two horizontal neighboring pixels would be shorted together, thus making $6 \mathrm{~mm} \times 12 \mathrm{~mm}$ pad). If a detector with smaller pixel would be chosen, we would still maintain the total pixel count the same by shorting more small pixels together horizontally where FDIRC optics is a simple pin hole camera, while maintaining small pixels in the y-direction where we do focusing.

One strong point of our design is that we share a total photon background load from a single bar box among $48 \mathrm{H}-8500$ detectors, and this results in acceptable rate even at the highest luminosity, and an acceptable total charge load after 10 years of operation. After adding shielding appropriately, a preliminary
MC simulation [9] indicates that the contribution from the active detector region inside the magnet is $\sim 50 \mathrm{kHz} /$ double-pixel, and a contribution from the photon camera, which is located outside of magnet, is $\sim 60 \mathrm{kHz} /$ double-pixel.

The final FDIRC electronics will do timing with pseudoCFD on a chip providing 100 ps resolution per channel, 1 $\mathrm{MHz}$ maximum background rate, $50 \mathrm{ps}$ double pulse resolution. There will also be an ADC measurement, allowing PMT monitoring and some improvement of the Cherenkov resolution with the charge sharing. The TDC parameters has $200 \mathrm{ps} /$ count, expected resolution of $\sim 100 \mathrm{ps}$ resolution, dead time at the input of $\sim 25 \mathrm{~ns}$, maximum rate (all channels firing) of $5 \mathrm{MHz} / \mathrm{channel,} \mathrm{maximum} \mathrm{rate} \mathrm{(1} \mathrm{channel} \mathrm{firing)} \mathrm{of}$ $20 \mathrm{MHz} / \mathrm{channel,} 1 \%$ dead time at $500 \mathrm{kHz}$ input rate on all channels, and $3 \%$ dead time at $1 \mathrm{MHz}$ input rate on all channels [3].

\section{A summary of R\&D results up to now}

It was very important to build a first FDIRC prototype with a single bar and oil-filled photon camera [10]. We learned how to operate new fast highly pixilated detectors [7], how to design a new optics, how to do the chromatic correction by timing (this was the very first RICH detector to achieve this). To be able to do such correction, one needs to achieve a timing resolution at a level of 200-250 ps per single photon, and the photon path length needs to be longer than 2-3 meters. The fact that FDIRC bars are longer (due to a penetration of the magnet iron) helps to improve the effect of this correction. We also learned that with $3 \mathrm{~mm}$ size pixels we could substantially improve on the FDIRC performance, and realized the role of photon path ambiguities on the Cherenkov angle tail [11].

\section{Expected performance of the final FDIRC}

Contributions to the Cherenkov angle resolution per photon and per track are shown in Table 1. Figure 8 shows the expected performance relative to the BaBar DIRC for various photodetector design choices. Figure 7 shows MC simulation of FDIRC expected Cherenkov angle resolution in CRT for H-8500 tube with $6 \mathrm{~mm}$ pixels and no chromatic correction. Clearly, a small pixel size and high QE efficiency is crucial to obtain the best possible performance, although a nominal design with $\mathrm{H}-8500$ tube and well-done chromatic corrections is not far back. The FDIRC performance with $\mathrm{H}-8500$ tube and no chromatic corrections would be approximately equivalent to the BaBar DIRC performance. At the end, we will make a conservative choice to make sure that the detector operation is smooth.

\section{Goals of the final FDIRC prototype test in CRT}

The FDIRC prototype will be tested at SLAC in CRT using 3D tracks. The tracking system has an angular resolution of $\sim 1.5 \mathrm{mrad}$, track position resolution of $\sim 5-6 \mathrm{~mm}$, start time resolution of better than $\sim 90 \mathrm{ps}$ (with tracking corrections it may reach $\sim 70 \mathrm{ps}$ ), a $1.6 \mathrm{GeV}$ muon low energy cut-off thanks 


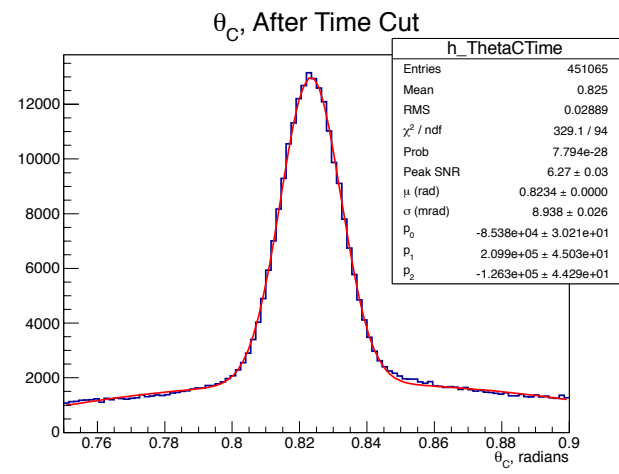

Figure 7: MC simulation of FDIRC expected Cherenkov angle resolution in CRT for $\mathrm{H}-8500$ tube with $6 \mathrm{~mm}$ pixels and no chromatic correction.

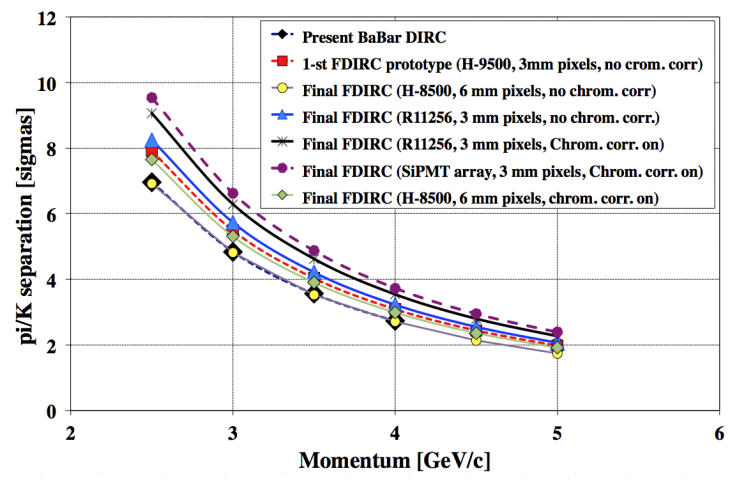

Figure 8: Final FDIRC expected PID performance for various detector options compared to the BaBar DIRC performance.

to a 46 inch-thick iron absorber, and a large iron area of 55 inch $\mathrm{x} 90$ inch allowing to reach large range of dip angles. The 3Dtracking capability is very important to understand tails of the Cherenkov angle distribution [11].

We will initially use SLAC amplifier and IRS2 electronics, which is a variant of waveform digitizing electronics [12]. Next year we will incorporate the final SuperB electronics [3].

The aim of the CRT test is to (a) verify that the new FDIRC optics works by measuring the Cherenkov angle resolution and its tail, and comparing it with the first FDIRC prototype results, (b) study a role of ambiguities on the Cherenkov angle tail, (c) study the chromatic error correction using 3D tracks, (d) study of several new detector options, and (e) study the FDIRC Cherenkov angle resolution and its tails by adding a random light noise while taking normal CRT data.

\section{Conclusion}

We have clearly demonstrated that the photon camera, made of solid fused silica, is build-able. Its main advantage is the radiation hardness, small size, stability and practically no maintenance. It is more expensive compared to oil-based or waterbased designs, but they require a long-term maintenance, which is at the end more expensive if one integrates over 10 years of operation. And fluid-based systems represent higher risk to other subsystems.

\begin{tabular}{|c|l|c|}
\hline Item & \multicolumn{1}{|c|}{ Cherenkov resolution per single photon } & {$[\mathrm{mrad}]$} \\
\hline 1 & Chromatic error & 5.5 \\
\hline 2 & Pixel contribution $(6 / 3 \mathrm{~mm}$ pixels) & $5.5 / 2.8$ \\
\hline 3 & Optical aberration & $1-9$ \\
\hline 4 & Transport along the bar & $2-3$ \\
\hline 5 & Bar thickness & $\sim 1$ \\
\hline 6 & Old wedge bottom inclined surface & 3.5 \\
\hline & Final error (no chromatic correction) & $10 / 8.8$ \\
\hline & Final error (with chromatic correction) & $8.4 / 7.0$ \\
\hline & \multicolumn{1}{|c}{ Cherenkov resolution per track } & {$[\mathrm{mrad}]$} \\
\hline Item & $\begin{array}{l}\text { BaBar DIRC } \\
\text { tion and H-8500 with QE } \sim 24 \%\end{array}$ & 2.5 \\
\hline 1 & $\begin{array}{l}\text { FDIRC with 6 mm pixels, chromatic correction } \\
\text { and H-8500 with QE } \sim 24 \%\end{array}$ & 2.2 \\
\hline 4 & $\begin{array}{l}\text { FDIRC with 3 mm pixels, chromatic correction } \\
\text { and R11256-M64 with QE } \sim 36 \%\end{array}$ & 1.9 \\
\hline
\end{tabular}

Table 1: FDIRC Cherenkov angle error contributions.

\section{Acknowledgments}

The authors would like to thank M. McCulloch for help in final assembly of the optics and prototype, M. Zago for the mechanical design of the Fbox and the Padova University mechanical workshop personnel for Fbox construction and assembly. We also thank M. Mongelli and V. Valentini of Bari for Fbox mechanical support structure design and M. Franco for helping to construct it. This work was supported in part by the Department of Energy, Contract DEAC02-76SF00515.

\section{References}

[1] B. Ratcliff, SLAC-PUB-5946, 1992, and I. Adams et al., Nucl. Instr. Meth. A583 (2007) 281.

[2] J. Va'vra, D. Roberts and B. Ratcliff, "FDIRC design for SuperB," Nucl. Instr. Meth. A404 (2011) 282.

[3] C. Beigbeder and D. Breton, "PID electronics for SuperB," this issue.

[4] J. Va'vra, "SLAC Cosmic Ray Telescope Facility," SLAC-PUB-13873, 2010.

[5] J. Va'vra, SLAC-PUB-13464, 2008, and "Focusing DIRC design for SuperB," SLAC-PUB-13763, 2009.

[6] D. Roberts, "Geant4 simulation of FDIRC," SuperB workshop, October 2009, http://agenda.infn.it/conferenceDisplay.py?confId=1742.

[7] C. Field et al., "Development of photon detectors for FDIRC," Nucl. Instr. Meth. A553, (2005) 96, and SLAC-PUB-11107, 2004.

[8] F. Gargano, "Study of H8500 MaPMT for the FDIRC detector at SuperB," this issue.

[9] A. Perez, "FDIRC background," SuperB workshop, June 2012, http://agenda.infn.it/conferenceDisplay.py?confId=4880.

[10] J. Benitez et al., "Status of the FDIRC," Nucl. Instr. Meth. A595, (2008) 104, and SLAC-PUB- 12236.

[11] K. Nishimura et al., "A detailed study of FDIRC prototype with waveform digitizing electronics in cosmic ray telescope using 3D tracks," to be published.

[12] L. L. Ruckman, G. S. Varner, and A. Wong, Nucl. Instr. Meth. A591, (2008) 534. 